\title{
Psychiatrie in der Medizin - Psychotherapie in der Medizin
}

\author{
Zuhören - Verarbeiten - In Beziehung sein - Intervenieren. Die Rolle der \\ Psychiatrie und Psychotherapie in der Medizin
}

\author{
Henriette Löffler-Stastka · Bettina Fink · Karin Matuszak-Luss · Reinhold Glehr $\cdot$ August Ruhs
}

Eingegangen: 20. Juli 2016 / Angenommen: 12. September 2016 / Online publiziert: 11. Oktober 2016 (c) Der/die Autor(en) 2016. Dieser Artikel ist eine Open-Access-Publikation.

Zusammenfassung Um dem Gegenstandsbereich der Psychotherapie in der Psychiatrie gerecht zu werden, gilt es die Subjektivität des Menschen zu betonen. Seit über 100 Jahren gilt die Zielsetzung der ersten psychotherapeutischen Tradition, der Psychoanalyse, sich nicht nur als therapeutische Methode zu verstehen, sondern auch in ihrer kulturtheoretischen Dimension, und sich vor allem in ihrer gesellschaftskritischen Aufgabenstellung immer wieder zu Wort $\mathrm{zu}$ melden.

In diesem Zusammenhang gilt es sich mit der Tatsache auseinanderzusetzen, dass gerade die Psychotherapie durch die Inklusion von geistes- und sozialwissenschaftlichen Forschungsfragen das naturwissenschaftliche Forschungsparadigma erweitert und ergänzt. Aus diesem Grund gestaltete die AG Ambulante Psychotherapie der Österreichischen Gesellschaft für Psychiatrie, Psychosomatik und Psychotherapie (ÖGPP) angesichts des Tagungstitels der ÖGPP-Jahrestagung 2015 „Psychiatrie in der Medizin“ in Gmunden einen Workshop zum Thema Psychotherapie in der Medizin und lud zur Diskussion ein. Wir stellten eine Literaturanalyse vor: Anhand von Thomas Bernhards Aussagen über „Die Medizin“, „Das Patiententum“, „Die Mediziner“ und „Der Medizinbetrieb“ wurde eine Abhandlung über das Subjektive im Menschen gezeigt, wobei diese Auseinandersetzung als Diskussions-Start diente, in der versucht wurde, den medizinischen Diskurs wieder gezielt mit

Für die AG Ambulante Psychotherapie der Österreichischen Gesellschaft für Psychiatrie, Psychosomatik und Psychotherapie (ÖGPP)

H. Löffler-Stastka ( $\bowtie)$

Klinik für Psychoanalyse und Psychotherapie, Medizinische Universitaet Wien, Waehringer Guertel 18-20, 1090 Wien, Österreich

henriette.loeffler-stastka@meduniwien.ac.at geisteswissenschaftlichen Diskursformen zusammenzubringen. Eine Auseinandersetzung als eine Übung, die nun schon beinahe vergessen worden war - und, um der Klage, dass über Ökonomie, Spezialisierung und Effizienzsteigerung, die Rolle der Ärztin/des Arztes als „Wesen an sich“ in der Gesellschaft, nicht mehr befriedigend reflektiert werden kann.

Um Thomas Bernhards Reflexionen und Anwürfe dem Leser näherzubringen und für die Aufnahme und Reflexion der Gedankengänge Offenheit zu erhalten, wurde auf folgende Werke Thomas Bernhards fokussiert: „Ein Kind“, „Die Kälte“, „Der Atem“ und „Frost“. Die Diskussion fokussiert Versorgungsaspekte (Karin Matuszak-Luss), gesellschaftliche Aspekte (August Ruhs), die Berufspolitik (Bettina Fink), die Ausbildung (Henriette Löffler-Stastka) und Aspekte der Kooperation (Reinhold Glehr). Im Folgenden werden Thomas Bernhards Positionen sowie deren mögliche Bedeutung für unser Fachgebiet der Psychiatrie als Anregung zur Reflexion erläutert.

Schlüsselwörter Naturwissenschaften · Geisteswissenschaften - Sozialwissenschaften - Psychiatrischepsychotherapeutische Versorgung · Ausbildung · Kooperation

\section{Psychiatry in medicine - psychotherapy in medicine \\ Listen - contain - engage - intervene. The role of psychiatry and psychotherapy in medicine}

Summary To give proper consideration to the field of psychotherapy in psychiatry, it is the subjectivity of the human being to emphasize. For over 100 years, the aim of the first psychotherapeutic tradition, psychoanalysis, was not only to function as a therapeutic method, but also to focus the cultural theoretical di- 
mension, and to argue especially in socio-critical tasks again and again.

In this context, it is to discuss the fact that psychotherapy expands research issues through the inclusion of humanities and the social sciences and completes the scientific research paradigm. For this reason the AG Outpatient Psychotherapy of the Austrian Society for Psychiatry and Psychotherapy (ÖGPP) created in view of the conference title of the ÖGPP Annual Conference 2015 "Psychiatry in Medicine" a workshop in Gmunden on "Psychotherapy in Medicine" and invited for discussion. We presented a literature analysis: Based on Thomas Bernhard's statements about "Die Medizin", "Das Patiententum", "Die Mediziner" und "Der Medizinbetrieb" we showed a treatise on the Subjective in Human being. This dispute served as a starting point for discussion, and we tried to specifically match the medical discourse with discourse forms of the humanities. A dispute as an exercise, which is almost forgotten now - and, to focus the complaint that due to economics, specialization and increase of efficiency, the role of the doctor as "human being itself" in society, no longer can be reflected satisfactorily.

To bring Thomas Bernhard reflections and accusations closer to the reader and to obtain openness for receiving and reflecting the thoughts, we focused on the following works of Thomas Bernhard: "Ein Kind", "Die Kälte", "Der Atem" und "Frost". The discussion then focused health care aspects (Karin MatuszakLuss), social aspects (August Ruhs), vocational policy (Bettina Fink), training (Henriette Löffler- Stastka) and aspects of cooperation (Reinhold Glehr). In this paper Thomas Bernhard positions and their possible implications for our field of psychiatry are described as suggestions for reflection.

Keywords Science - Humanities - Social Sciences • Psychiatric-psychotherapeutic care - Psychiatric training $\cdot$ Cooperation

Versorgungsaspekte der Fachärzte und Fachärztinnen für Psychiatrie und psychotherapeutische Medizin - mit Bezug auf "Ein Kind." (Thomas Bernhard, 1982) (Bernhard 2010)

Ein Kind von Thomas Bernhard ist u. a. ein Lehrbeispiel für eine multiple - komplexe Traumatisierung. In diesem Roman mit stark autobiografischen Zügen berichtet der Erzähler, ein Kind, von den seelischen und körperlichen Misshandlungen durch seine Umgebung vor und während des zweiten Weltkrieges. Thomas Bernhard skizziert mit diesem Werk eine komplexe multiple Traumatisierung, die durch eine verstörte Mutterbindung, durch Traumatisierungen in der Schule, durch den destruktiven Umgang mit dem Bettnässen des Kindes und durch Kriegstraumatisierungen verursacht wird.
Anhand von Zitaten aus dem Roman werden Introjektbildungen und Ego States (Watkins und Watkins 2008) des Kindes aufgezeigt, in welchen Theorie und Therapie zwischen grundsätzlich ressourcenreichen Ego States, verletzten Ego States und verletzenden, destruktiv wirkenden Ego States unterschieden werden können. Im Folgenden werden für jeden der genannten Ego States Romanzitate exemplarisch angeführt:

- Beispiel für ressourcenreichen Ego State: (Bernhard 2010, S. 24): „Zum Großvater nach Ettendorf ging ich, wie immer, auch in dieser Nacht wie auf einen heiligen Berg hinauf.“ Oder S. 93/94: „Ich träumte von meinen zukünftigen Reisen ... Während des Unterrichts sah ich immer mehr in die Wolkenkratzerschluchten von Manhattan als auf die Tafel vor mir, auf welcher der Lehrer mathematische Öde ausbreitete.“

- Beispiel für traumatisierten Ego States: (Bernhard 2010, S. 49): „Vor dem Ochsenziemer hatte ich Angst, aber die Schläge, die meine Mutter mir damit zufügte, hatten keine Wirkung. Mit den teuflischen Wörtern erreichte sie ihr Ziel, dass sie Ruhe hatte, andererseits stürzte sie mich damit jedesmal in den fürchterlichsten aller Abgründe, aus welchem ich dann zeitlebens nicht mehr herausgekommen bin. Du hast mir noch gefehlt! Du bist mein Tod! In den Träumen werde ich noch heute damit gepeinigt.“

- Beispiele für destruktive Ego States: (Bernhard 2010, S. 14): „Ich liebte meine Mutter, aber ich war ihr kein lieber Sohn, nichts war einfach mit mir, alles Komplizierte meinerseits überstieg ihre Kräfte. Ich war grausam, ich war niederträchtig, ich war hinterhältig, ich war, das war das Schlimmste, gefinkelt. Der Gedanke an mich erfüllte mich mit Abscheu." (Täterintrojekt - feindliche Introjektion).

Fazit aus der Diskussion war, dass es u. a. auch die Aufgabe der Fachärzte und FachärtzInnen für Psychiatrie ist, Allgemeinmediziner und Vertreter anderer somatisch-medizinischer Bereiche in Bezug auf psychiatrische Erkrankungen zu sensibilisieren, was einen Versorgungsaspekt darstellt.

Dort, wo PatientInnen wechselnde klinisch-somatische Befunde zeigen, die nicht wirklich auf somatische Therapieinterventionen reagieren, liegt der Verdacht auf eine psychische Komponente nahe. Dann heißt es sich Zeit für den/die PatientIn nehmen, die Lebenshintergründe genauer anzusehen. Ein Umstand, der von den Krankenkassenträgern zurzeit nicht gewünscht und gefördert wird.

Entsteht ein Verdacht auf eine schwerwiegende biografische Belastung (u. a. Traumata) steht der/die somatisch orientierte Arzt/Ärztin vor der Aufgabe die PatientInnen zur Psychotherapie und psychiatrischen, fachärztlichen Behandlung zu motivieren. 
Gesellschaftliche und kulturelle Aspekte - mit Bezug auf "Der Atem. Eine Entscheidung." (Thomas Bernhard, 1978) (Bernhard 2014)

Wenn Freud von den Dichtern als psychoanalytischen Bundesgenossen spricht, weist er auf deren besondere Sachverständigkeit in der Seelenkunde hin, weil sie aus Quellen schöpfen würden, welche man noch nicht für die Wissenschaft erschlossen habe (Freud 1907, S. 14).

Allerdings erstreckt sich die Sensibilität von Literaten (und anderen kreativen Menschen) auch auf die subtile Beschreibung körperlicher Krankheiten und ihrer psychischen Verarbeitung, so dass wir über eine Fülle an Krankengeschichten zweiter Ordnung verfügen, die eine wertvolle Ergänzung zur nüchternen Objektivität des medizinischen Diskurses und des ärztlichen Jargons darstellen.

1949 erleidet Thomas Bernhard eine offenbar verkühlungsbedingte „nasse Rippenfellentzündung“, an deren Stelle sich im weiteren Krankheitsverlauf eine Lungentuberkulose als Hospitalismuseffekt setzt. In Anbetracht der Todesnähe im ersten Abschnitt seines Leidens und unter dem Eindruck des Sterbezimmers, in das man ihn verlegt hat, entscheidet er sich nicht nur für das Leben und gegen den Tod, sondern er besinnt er sich auf die Worte seines Großvaters, der den kreativ-sensiblen Kranken und Betroffenen eine besondere Kompetenz bezüglich der Erfassung, der Entfaltung, des Verlaufs und des Ausgangs einer Krankheit bescheinigt:

Der Kranke ist der Hellsichtige, keinem anderen ist das Weltbild klarer. Wenn er die Hölle, so hatte er fortan das Krankenhaus bezeichnet, verlassen habe, seien die Schwierigkeiten, die es ihm in letzter Zeit unmöglich gemacht hätten, zu arbeiten, beseitigt. Der Künstler, insbesondere der Schriftsteller, hatte ich von ihm gehört, sei geradezu verpflichtet, von Zeit zu Zeit ein Krankenhaus aufzusuchen, gleich, ob dieses Krankenhaus nun ein Krankenhaus sei oder ein Gefängnis oder ein Kloster. Es sei das eine unbedingte Voraussetzung. Der Künstler, insbesondere der Schriftsteller, der nicht von Zeit zu Zeit ein Krankienhaus aufsuche, also einen solchen lebensentscheidenden existenznotwendigen Denkbezirk aufsuche, verliere sich mit der Zeit in die Wertlosigkeit, weil er sich in der Oberflächlichkeit verheddere. Dieses Krankenhaus, so mein Großvater, kann ein künstlich geschaffenes Krankenhaus sein, und die Krankheit oder die Krankheiten, die diesen Krankenhausaufenthalt ermöglichen, können durchaus künstliche Krankheiten sein, aber sie müssen da sein oder müssen erzeugt und müssen immer unter allen Umständen in gewissen Abständen erzeugt werden. Der Künstler oder der Schriftsteller, der sich um diese Tatsache herumdrücke, gleich, aus was für einem Grund, sei von vornherein zur Wertlosigkeit verurteilt. (Bernhard 2014, S. 48 f.)

Einigermaßen genesen soll der achtzehnjährige Bernhard seine Krankheit in einem Erholungsheim in
Großgmain ausklingen lassen. Stattdessen „holt“ er sich dort seine Tuberkulose und damit den stigmatisierenden Status eines „Lungenkranken“, wodurch er auch mit bestimmten gesellschaftlichen Dimensionen des Krankseins und sozial unterschiedlichen medizinischen Dispositiven konfrontiert ist.

Jetzt, während der Mahlzeit, die ich nur unter äußerster Anstrengung, in meinem Bett sitzend, einnehmen hatte können, war Gelegenheit gewesen zu einer ersten Unterhaltung mit meinem Mitpatienten. Er war schon die dritte Woche in diesem Zimmer und glaubte, nach drei weiteren Wochen nach Hause gehen zu können. Er war, genau wie ich, aus der Ersten Internen, wie er sich ausdrückte, aber schon drei Wochen früher hierher gebracht worden. Er war, zum Unterschied von mir, ein Klassepatient und im Krankenhaus, im Unterschied zu mir, der ich in einem Zimmer mit sechsundzwanzig Betten untergebracht gewesen war, in einem Zweibettzimmer gelegen, und was er aus dem Krankenhaus berichtete, war allein dadurch vollkommen anders, ja in vielen, in den meisten Punkten geradezu entgegengesetzt dem, das ich berichtete, seine Erlebnisse waren vollkommen andere, wie auch die Ereignisse, die er erlebt hatte, vollkommen andere gewesen waren als die meinigen, denn er war die ganze Zeit mehr oder weniger von allen Geschehnissen und Ereignissen, die ich erlebt hatte, abgeschirmt gewesen durch die Tatsache, daß er, als Klassepatient, in einem Zweibettzimmer gelegen und durch diesen Vorzug von vornherein mit der tagtäglichen Masse der Fürchterlichkeit und des Schreckens in diesem großen Krankenhaus überhaupt nicht in Berührung gekommen war. Der Klassepatient, wenn er allein liegt, hat nur seine eigenen Leiden zu leiden, seine eigenen Schmerzen auszuhalten, und seine Beobachtung beschränkt sich auf die Beobachtung seiner eigenen, kranken Person und auch nur auf die Umwelt und Umgebung seiner eigenen, kranken Person, während der andere, der kein Klassepatient ist, in seine eigenen Leiden und in seine eigenen Schmerzen und in die Beobachtung der eigenen, kranken Person die Leiden und Schmerzen und die Beobachtung auch all jener einzubeziehen hat, die mit ihm sein Zimmer zu teilen haben, und im Falle meines neuen Zimmergenossen war es nur ein einziger anderer gewesen, in meinem Fall aber waren es fünfundzwanzig. [...] So musste, was ich aus dem Krankenhaus zu berichten gehabt hatte, naturgemäß etwas vollkommen anderes sein als das, was der Architekturstudent berichtete. Aber damit ist nicht gesagt, dass die Erlebnisse meines Mitpatienten, mit welchem ich mich sehr schnell angefreundet hatte, weniger tief auf ihn eingewirkt hätten als die meinigen auf mich und ihn weniger verletzt und verstört und zerstört hätten. Aber die Perspektive des sogenannten Klassepatienten ist naturgemäß immer eine andere als die des sogenannten gewöhnlichen, einfachen, welcher niemals auch nur das Geringste zu fordern hat und dem letzten Endes, zum Unterschied vom Klassepatienten, nichts erspart bleibt, weil er nicht, wie der Klassepatient, in jedem Augenblick und bei jeder Gelegenheit 
auf irgendeine, wenn auch noch so unscheinbare Weise geschont und geschützt und abgeschirmt wird, während jener in den meisten Fällen ja doch niemals zu dem Blick in das äußerste Hässliche und in das größte Entsetzen gezwungen ist. Dem Klassepatienten ist alles abgeschwächt, herabgemildert, es wird ihm, zum Unterschied von den anderen, nicht alles und immer wieder alles mit der größten Rücksichtslosigkeit zugemutet. Inzwischen hat sich auch in unserem Land auf diesem Gebiet viel geändert. Noch sind die Klassen in den Krankenhäusern nicht abgeschafft, aber wir müssen darauf bestehen, dass sie abgeschafft werden, und zwar so bald als möglich abgeschafft werden, weil die Tatsache, dass es ausgerechnet in den Krankenhäusern noch immer Klassen gibt, tatsächlich ein menschenunwürdiger Zustand, eine gesellschaftspolitische Perversität ist. (Bernhard 2014, S. $98 \mathrm{ff}$.).

Was in diesen kurzen Textpassagen Thomas Bernhards als pars pro toto zum Ausdruck kommt, ist eine feinsinnige Analyse der subjektiven Dimensionen eines Krankheitsverlaufs. Sie umfasst die seelischen $\mathrm{Zu}-$ stände und Erschütterungen eines von einer schweren Krankheit heimgesuchten Menschen, beschreibt die inneren Kämpfe, die sich sowohl gegen das Leiden selbst als auch gegen den medizinischen Umgang damit beziehen und scheut auch nicht vor der Kritik an einer Ungleichbehandlung von Betroffenen hinsichtlich ihres unterschiedlichen sozialen Status und ihrer finanziellen Möglichkeiten zurück.

Gerade in einer Gegenwart, die man häufig als posthumanistisch bezeichnet, ist auch das medizinische Denken vorwiegend naturwissenschaftlich und technologisch determiniert, was sich unter anderem durch die Reduktion des Subjekts auf sein Gehirn, durch eine weitgehende Parallelisierung von Mensch und Tier und durch eine Konzentration auf genetische Faktoren gegenüber der Beachtung umweltbedingter Kausalitäten äußert.

Dabei ist für alles, was in den Erkenntnisbereich Mensch fällt und eine Wissenschaft vom Menschen begründet, die Berücksichtigung geistes- und sozialwissenschaftlicher Perspektiven und Paradigmen eine unerlässliche Bedingung. Für die Humanmedizin bedeutet dies, dass sie die an der Krankheit gewonnenen objektiven Daten und Fakten stets in den Erlebenskontext des betroffenen Kranken integrieren muss, sofern sie auf die Aufrechterhaltung ihrer Grenzen gegenüber der Veterinärmedizin bedacht bleiben will.

Die Medizingeschichte sagt uns, dass ärztliches Denken und Handeln bis etwa zur Mitte des vorigen Jahrhunderts durchaus von einer solchen ganzheitlichen Auffassung menschlichen Krankseins geprägt war, wodurch die Einstellung des Patienten sowohl zu seiner Krankheit als auch zur Person seines Arztes und den von ihm verordneten Medikamenten bzw. den von ihm gesetzten Maßnahmen im Behandlungsprozess mitberücksichtigt wurden. Mit der industriellen Revolution sei es aber zunehmend $\mathrm{zu}$ einer Loslösung der objektiv erfassbaren Krankheit von ihren betroffenen Subjekten gekommen, sodass sich ein objektiv-technologischer Medizinbereich von einem subjektiv-psychologischen Bereich trennte und schließlich immer mehr auf zwei voneinander unabhängig werdende Kompetenzen verteilt wurde.

Man sollte sich mehr bewusst sein, dass durch die Herausbildung eines eigenen psychotherapeutischen Berufsstandes, aber auch durch die Entwicklung alternativmedizinischer Modelle diese Kompetenzentrennung in der Medizin aufrechterhalten wird, sodass es für das medizinische Denken und Handeln mehr denn je notwendig ist, sich dieses psychologisch-psychotherapeutische Terrain wieder verstärkt anzueignen, um sich nicht dem alten Vorwurf ausgesetzt zu fühlen, dass Ärzte und ärztliches Personal nur Handlanger einer übermächtigen Medizinindustrie darstellen würden.

Das Schwergewicht der Subjektivität auch in den Bereichen von Gesundheit und Krankheit erklärt sich daraus, dass gerade das Subjektive und je Besondere das spezifisch Menschliche in diesem komplexen Geschehen darstellt. Da der Mensch die unvermittelte Beziehung zur Natur im Laufe seiner phylo- und ontogenetischen Entwicklung verloren hat, da er über Instinkte im eigentlichen Sinn nicht verfügt, muss er sein ganzes Leben einschließlich seiner sexuellen und nicht-sexuellen Bedürfnisse über kulturell-gesellschaftliche und damit sehr freie und relativierbare Normensysteme regeln.

Man hat zwar den Terminus der „spezifischmenschlichen Krankheiten“ geprägt, um jene Krankheiten zu kennzeichnen, die der Mensch nicht mit dem Tier gemeinsam hat, bei deren Entstehung also soziale, kulturelle und psychische Faktoren eine ganz wesentliche Rolle spielen, läuft dabei aber Gefahr, eine unangemessene Polarisierung zwischen rein somatischen, d. h. zoologisch-anthropologischen Krankheiten einerseits und den Psychoneurosen andererseits aufzustellen. Wenn man aber bereit ist, bei allen Krankheitserscheinungen stets ein davon betroffenes Subjekt mitzudenken, so erscheint insofern jede menschliche Krankheit als spezifisch menschlich, als sie an ein Bewusstsein gebunden ist, das dieser Krankheit Bedeutung verleiht. So könnte man sagen, dass Krankheiten ihre spezifisch menschliche Dimension als Vorstellung mit entsprechender affektiver Färbung nur in Zuständen tiefer Bewusstlosigkeit des Kranken verlieren, sodass sie dann nur noch in ihren objektiven Aspekten zur Erscheinung gelangen. Es ist aber nicht auszuschließen, dass selbst in komatösen Zuständen ein psychischer Basisapparat funktionieren dürfte, welcher noch Eindrücke der Innen- oder Außenwelt des Patienten aufnehmen und verarbeiten könnte.

Unter dieser Perspektive stellen Psychiatrie, Psychotherapie und medizinische Psychologie, die weitgehend den Sozial- und Geisteswissenschaften verhaftet sind, privilegierte Disziplinen dar, um die Prinzipi- 
en einer tatsächlichen Humanmedizin offenzulegen. Damit tritt auch neben der „Behandlungstechnik“ medizinisches Handeln als „Heilkunst“ stärker in den Vordergrund. Damit sind die Bedürfnisse des kranken Körpers und die Ansprüche des Patienten stets im Zusammenhang mit den zum Teil unbewussten Begehrenshaltungen des kranken Subjekts zu sehen.

Wenn man eine Krankheit immer unter den drei Aspekten: somatischer Zustand, ärztliche Interpretation und bewusste und unbewusste seelische Verfasstheit des Kranken betrachtet, so gilt im Allgemeinen, dass das Bewusstsein eines Patienten von einem Leiden und einem Gefühl der Schwäche sowie von Angst vor etwaigen Folgen sowohl der Krankheit als auch der Behandlung geprägt ist. Dies im Gegensatz zur Phänomenologie der Gesundheit, die durch ein Gefühl der Sicherheit und des Lebensvertrauens bestimmt wird. Im Krankheitsfall oder vor einer Festlegung desselben haben Diagnose und Prognose eines Arztes für einen tatsächlichen oder potentiellen Patienten zwei gegensätzliche Funktionen: sie sind sowohl mit einem Moment der Gefahr als auch mit einem Gefühl der Hoffnung und der Zuversicht verbunden. Immer aber ist mit einem Widerstand gegen die Annahme einer Krankheit und gegen die Akzeptierung einer Patientenrolle festzustellen, der seine Motivationen aus verschiedenen Quellen schöpft.

Die Lösung dieses Konflikts entscheidet schließlich über die Annahme einer Patientenrolle und über die Konsultation eines Arztes oder einer medizinischen Einrichtung.

Wenn auch unbewusste Verarbeitungen sowohl primär psychogener als auch primär somatischer Krankheiten weder ohne weiteres erkennbar noch unzulässig verallgemeinerbar sind, da sie der intimsten und privatesten Sphäre eines Patienten angehören und somit immer in gewissem Maß einzigartig sind, so liegt doch ein gemeinsamer Zug aller von einer Krankheit Betroffenen darin, dass jede Krankheit mit Regressionsphänomenen der Persönlichkeit einhergeht. Hilfsbedürftigkeit bis zur Hilflosigkeit sowie der mit narzisstischer Einengung einhergehende Abzug libidinöser Besetzungen äußerer Objekte stehen dabei im Vordergrund.

Nicht selten finden wir im Gefolge einer körperlichen Krankheit psychische Desintegrations- und Dekompensationserscheinungen mit einem zumeist vorübergehenden Verlust eines vorher bestehenden labilen seelischen Gleichgewichtes. Andererseits finden wir in der Anamnese von psychotisch Erkrankten oftmals körperliche Erkrankungen oder Traumen auch nur geringen Ausmaßes, welchen zumindest eine mitauslösende Funktion für eine psychotische Dekompensation zugeschrieben werden kann. Selbstverständlich kann auch in manchen Fällen die Bedrohung der körperlichen und seelischen Desintegration zu einem Ausagieren der Phantasmen führen, was dann in suizidalen oder homizidalen Handlungen ein katastrophales Ende finden kann. Denn es ist zu bedenken, dass narzisstische Erlebnisstrukturen mit ihrer Entfremdungs- und Auflösungsgefahr immer Aggressivität als korrelative Spannung mit sich führen, welche sich dann zu den libidinösen Selbst- und Selbstobjektbesetzungen hinzugesellen.

Regressionsbewegungen auf dieses Niveau sind aber auch immer mit magischem Denken verbunden, was einerseits mit irrealen Vorstellungen gegenüber dem Arzt einhergeht und dessen besonderen gesellschaftlichen Status mitbedingt und was andererseits zu religiösen und abergläubischen Einstellungen gegenüber der Krankheit und allen möglichen sie beeinflussenden Personen, Mächten, Handlungen und Maßnahmen führt.

Grundsätzlich lässt sich sagen, dass jede Krankheit kränkt und dass auf einem höheren psychischen Niveau der Betroffene nicht nur mit Angst, sondern auch mit Scham und Schuldgefühlen auf die Erkrankung reagiert.

Bei der subjektiven Verarbeitung von Krankheit ist weiterhin das $\mathrm{zu}$ beachten, was man als die private Anatomie und den hysterischen Körperatlas des Patienten bezeichnen kann. Jenseits ihrer wissenschaftlichen Bedeutung, welche vor allem für den Arzt relevant ist, ist jede Körperregion und jedes Organ konnotativ mit einem Bedeutungshof umgeben, welcher abgesehen von persönlichen und intimen Erfahrungen zum Teil auch jenen allgemeinen emotionalen Zuschreibungen entstammt, wie sie die Alltagssprache und der Volksmund den verschiedensten Organen verleiht. Dieser Umstand, für den man Beispiele wohl nicht zu nennen braucht, führt uns in den Problembereich der Psychosomatik, weil es dort um Phänomene geht, in welchen Erlebnisse und Erfahrungen in einer Verflechtung von unmittelbaren körperlichen Reaktionen mit primären und undifferenzierten psychischen Vorstellungen das Individuum erfassen. Ein anderer großer Teil an Organsymbolik resultiert wiederum aus rein privaten Phantasiebildungen, welche das Ergebnis von traumatisierenden Fehlinterpretationen und semantischen Missverständnissen in Bezug auf Bedeutungen und Funktionen von Körperteilen sind.

Was nun den Zugang zu solchen zum Teil unbewussten subjektiven Krankheitsverarbeitungen betrifft, ist festzustellen, dass es nicht so sehr darauf ankommt, dass der Arzt mit dem Patienten spricht, sondern dass er ihm zuhört. Hier geht es also primär nicht so sehr um eine Aufklärung des Patienten durch den Arzt, sondern umgekehrt, um eine Aufklärung des Arztes durch den Patienten. Denn was ein Organ, ein Symptom oder eine Krankheit für einen Arzt bedeutet, ist zumeist etwas anderes, als es für den Patienten bedeutet. Für diesen geht es vor allem um private und intime Vorstellungen und Phantasien, für die er sich schämt, bei denen er vielleicht Schuld empfindet, vor denen er sich fürchtet. Und dies in besonderem Maß vor der Autorität des Arztes. Dessen Zuhören muss daher von besonderer Art sein, weil es die Wahrnehmung mehrerer Sinnesqualitäten miteinzuschließen 
hat, um den Kranken dort zu verstehen, wo er etwas nicht mehr sagen kann.

\section{Berufspolitische Aspekte - mit Bezug auf „Die Kälte. Eine Isolation." (Bernhard 1985)}

Mit der Einweisung in die Lungenheilstätte Grafendorf bei St. Veit beginnt ein neues Kapitel der Leidensund Lebensgeschichte des 18-Jährigen Bernhard. Ein Schatten auf der Lunge verbannt ihn in die isolierte Welt des Sanatoriums, aus der es so leicht kein Entrinnen gibt. Thomas Bernhard Schilderungen von entsetzlichen Erlebnissen des Ausgeliefertseins in einer Welt der Hoffnungslosigkeit, Isolation, Beziehungs-und Sprachlosigkeit, in der Patienten Opfer einer selbstherrlichen Anstaltsleitung werden, stellt Ausgang und Vergleich für einige kritische Reflexionen aus berufspolitischer Sicht dar.

Mit dem sogenannten Schatten auf meine Lunge war auch wieder ein Schatten auf meine Existenz gefallen. Grafenhof war ein Schreckenswort, in ihm herrschten absolut und in völliger Immunität der Primarius und dessen Assistent und dessen Assistent und die für einen jungen Menschen wie mich entsetzlichen Zustände einer öffentlichen Lungenheilstätte. Hilfe suchend, bin ich doch hier mit nichts als Hoffnungslosigkeit konfrontiert gewesen, das hatten schon die ersten Augenblicke, ersten Stunden, noch unerhörter die ersten Tage gezeigt. Die Lage der Patienten verbesserte sich nicht, sie verschlimmerte sich mit der Zeit, auch meine eigene, ich fürchtete, hier genau denselben Weg gehen zu müssen wie die vor mir nach Grafendorf Eingewiesenen, an welchen ich nichts als Trostlosigkeit ihrer Verfassung ablesen, an welchen ich nichts anderes als den Verfall studieren konnte. Auf meinem ersten Weg in die Kapelle, in welcher täglich eine Messe zelebriert worden ist, hatte ich ein Dutzend Partezettel an den Wänden zu lesen bekommen, lakonische Texte über in den letzten Wochen Verstorbene, die, so mein Gedanke, gerade noch wie ich durch diese hohen kalten Gänge gegangen waren. (Bernhard 1985, S. 7)

Wie aus Bernhards Texten hervorgeht, findet Diagnostizieren und Behandeln ohne Interesse am subjektiven Leid und ohne Beziehungsaufnahme durch die Ärzte und das Pflegepersonal statt. Das Leiden an der Isolation ist so groß, dass Bernhard in pervertierter Weise den positiven Lungenbefund mit Sehnsucht erwartet, da dieser ihm die Zugehörigkeit zur Schicksalsgemeinschaft ermöglicht.

„In Anbetracht meiner leeren Spuckflasche hatte ich das bedrückende Gefühl, versagen zu müssen, und ich steigerte mich mehr und mehr in einen absoluten Auswurfwillen, in eine Auswurfhysterie hinein". (Bernhard 1985, S. 11)

„Die Verachtung traf mich zutiefst. Alle waren ansteckend, also positiv, ich nicht. (...) Zuerst waren, schien mir, nur die Ärzte enttäuscht, schließlich ich selbst.“ (...) „Nach fünf Wochen war es soweit, der Befund war: positiv. Ich war plötzlich Vollmitglied der Gemeinschaft."(Bernhard 1985, S. 13)

In dem von Bernhard eindrucksvoll beschriebenen Leiden, das iatrogen und durch die sozialen und institutionellen Pathologien zur Unerträglichkeit gesteigert wird, drängen sich gewisse Parallelen zum Leiden unserer PatientInnen aufgrund der Entwicklungen in unserem Gesundheitssystem auf.

In der Gesundheitspolitik lässt sich mit Besorgnis eine Entwicklung beobachten, die unter dem Diktat der Ökonomie und Rationalität, d.h. der Effizienz, Vorhersagbarkeit, Kontrolle und quantitativen Erfassbarkeit zu einem Verlust eines ganzheitlichen Behandlungszugangs führt. Letzterer ist aber nun gerade für unser Fach paradigmatisch, indem es um ein Verstehen der Erkrankung in Zusammenhang mit den sozialen Beziehungen, der Lebensgeschichte und den unbewussten Konflikten geht. Die ganzheitliche Auffassung führt dazu, dass eine Behandlung mit dem Verschwinden der Symptome nicht automatisch für abgeschlossen betrachtet wird, sondern bedacht wird, dass die den Symptomen zugrundeliegenden Konflikte erst zugänglich werden, in der Übertragung oder in anderen sozialen Beziehungen, d. h. als Beziehungskonflikte. Diese Phase der Bearbeitung oder des Durcharbeitens ist notwendig, um symptomatische Verbesserungen zu stabilisieren. Das Interesse der Kostenträger hingegen reduziert sich auf die Kontrolle von außen und die möglichst frühzeitige Beendigung der Behandlung unter isolierter Berücksichtigung der Symptome und der Funktionalität, wie der Arbeitsfähigkeit. Die Kostenträger greifen zunehmend in den psychotherapeutischen Prozess in nicht selten destruktiver Weise ein, indem sie entgegen der Indikationsstellung der BehandlerInnen notwendige Behandlungsfrequenzen reduzieren, die Behandlungsdauer vorzeitig determinieren oder die Behandlung ganz verwehren. Dem Patienten, der es wagt sich aus der Isolation seines Leidens im Kampf gegen Schuld-, Schamgefühle und Ängsten vor Abhängigkeit in Behandlung $\mathrm{zu}$ begeben, wird von Chefärzten und GutachterInnen in ihrem Furor von Kosten- und Therapiekontrolle erklärt, dass seine Krankheit keiner Behandlung bedürfe, er sich lediglich vom Therapeuten abhängig mache und es genüge Medikamente zu nehmen. Die Stigmatisierung der Tuberkulose damals ist übrigens mit der von psychischen Krankheiten heute durchaus vergleichbar. Dies führt zu einer Sprachlosigkeit des leidenden Patienten, einer Isolation des leidenden Subjektes, das auf die Kommunikation von Symptomen reduziert wird, wie sie uns Bernhard in erschreckender Weise vor Augen führt. Ein Verstehen der den Symptomen zugrundeliegenden Konfliktdynamik und der inneren Welt der Objektbeziehungen wird so verunmöglicht. Die Nachhaltigkeit der Veränderungen korreliert ja, wie wir aus der Psychotherapieforschung wissen, mit 
der Behandlungsdauer. Gerade psychiatrische Patienten, deren Beziehungserfahrungen zumeist von mangelnder Objektkonstanz und fehlenden verlässlich guten Objektbeziehungen geprägt sind, treffen auf ein Versorgungssystem, das Beziehungsabbrüche strukturell und systematisch vorsieht, welche dadurch legitimiert werden, dass sie zum Konzept erklärt werden.

Trotz der Macht der Verhältnisse gibt die bisherige berufspolitische Arbeit Anlass zur Hoffnung. Solidarisierung und Mobilisierung von mitbetroffenen BehandlerInnen, welche wie ihre PatientInnen aus der Position der Isolation und Ohnmacht gebracht werden müssen um sich gegen unqualifizierte und stigmatisierende Behandlungsverweigerer zur Wehr setzen zu können, ist erforderlich.

„Der Kranke muss sein Leiden selbst in die Hand und vor allem in den Kopf nehmen gegen die Ärzte“. (Bernhard 1985, S. 23)

So wie es Thomas Bernhard nach anfänglicher Selbstaufgabe durch seine Beziehung zu Musikern und Schriftstellern gelungen ist - in ihrer inneren Repräsentanz als gutes Gruppenobjekt, das ihm half innere Stärke oder Resilienz zu entwickeln - sich aus der krankmachenden Anstalt zu befreien und zu genesen, kann die Gruppe von Widerstand leistenden Kollegen und Kolleginnen auch durch ihre innere Repräsentanz letztlich ihren PatientInnen helfen sich zur Wehr zu setzen.

\section{Aus- und Weiterbildungsaspekte mit Bezug auf „Frost“ (Bernhard 1963):}

Was ist nun hier die Aufgabe der Psychotherapie in der Medizin?

Die Situation der Medizin-Ausbildung (prä- und postgraduell) wurde anhand des Werkes „Frost“ beleuchtet: „Frost“ war Thomas Bernhards erster literarischer Erfolg. Seine Radikalität ist verstörend und faszinierend. Ein Medizinstudent absolviert in einer chirurgischen Abteilung eines Provinzspitales eine Famulatur und im Rahmen der klinischen Arbeit bekommt er die Aufgabe, den Kunstmaler Strauch zu beobachten, ihn bei Spaziergängen zu begleiten und zuzuhören. Im Roman Frost führt der Medizinstudent 26 tagelang Tagebuch über seine Begegnungen mit dem Maler Strauch. Der Erzähler ist hier der Medizinstudent, der Roman eröffnet mit drastischen Szenen:

„Eine Famulatur besteht nicht nur (...) aus Bauchfellaufschneiden, Lungenflügel zu klammern und Fußabsägen, (...) Nicht nur daraus, dass ich sage: „Es wird schon!"- wo nichts mehr wird. (...) Mein Auftrag, den Maler Strauch zu beobachten, zwingt mich, mich mit solchen außerfleischlichen Tatsachen und Möglichkeiten auseinanderzusetzen. Etwas Unerforschliches zu erforschen. (...) es kann durchaus sein, dass das Außerfleischliche, nämlich das ohne die Zellen, das ist, woraus alles existiert, (...)“.
„Frost“ behandelt auch die Auseinandersetzung mit der Wissenschaft in folgendem Maße:

„Wir kennen jetzt die Naturgesetze (...). Wir sind von der Klarheit, aus welcher unsere Welt plötzlich ist, unsere Wissenschaftswelt, erschrocken; wir frieren in dieser Klarheit; aber wir haben diese Klarheit haben wollen, heraufbeschworen (...). Mit der Klarheit nimmt die Kälte zu. (...)"

„Wie das Gehirn plötzlich nur mehr Maschine ist, wie es noch einmal alles exakt herunterhämmert, (...) Als zöge ein zwergenhafter Diktator (...), an einem ungeheuren Mechanismus, der alles und alles in Gang setzt, in fürchterlicher und verheerender Lärmentwicklung, gegen die man aber nicht vorgehen kann ... “

Gegen Ende des Romans kommt der Studierende zu einem Ergebnis, einer Komposition, Thomas Bernhard eröffnet im anarchischen Spiel sprachlogischer Dekonstruktionen einen neuen Zwischenraum. Es ist Thomas Bernhards musikalischer Rettungsversuch vor der Herrschaft instrumenteller Vernunft. Thomas Bernhardts literarische Relativitätstheorie.

Ich habe, glaube ich, meine wissenschaftliche (...) Erforschungsmethode gefunden, einen Weg der Entdeckungen, einen solchen der nebeneinander-ineinanderuntereinander verlaufenden, miteinander korrespondierenden Anschauungsmöglichkeiten (...).

Und genau dies ist die Aufgabe der Psychotherapie in der Medizin, die „Musik“ jedes Einzelnen aufzunehmen und herauszuarbeiten, was auch immer die Komposition für jeden Einzelnen bedeutet, in welch hoffnungslos gestalteten Situation auch immer der Kranke sich befindet, und auch jeder körperlichen Regung oder jedem affektivem Ereignis eine Bedeutung zu geben. Hier können wir Psychoanalytiker und Psychotherapeuten das meiste einbringen, wenn wir uns beispielsweise das Konzept der „Geburt des Menschen“ vergegenwärtigen (Fonagy et al. 2002). Das Konzept zur mentalisierten Affektivität geht davon aus, dass jeder Mensch affektive Kurzereignisse und körperliche Sensationen erlebt, diese als Signal oder als eine Aussage an einen Therapeuten sendet; dieser kann resonant auf den Affekt eingehen und durch seine Reverie, Alphafunktion (Bion 1967) oder Reflective Functioning (Fonagy et al. 2002). Aufgrund dieser Denkfähigkeit und Reflexionsfähigkeit kann eine adäquate Reaktion gesetzt werden, die es dem Patienten ermöglicht eine höhere Ordnung, eine psychologische Ordnung, also eine Bedeutung zu einer körperlichen Ebene zu entwickeln. Dies ist die Hauptaufgabe der Psychotherapie in der Medizin.

Was können wir nun für die Ausbildung dieser Kompetenzen tun?

Wenn wir dem Nobelpreisträger Daniel Khanemann folgen, empfiehlt er zwei Arten von Denken: „thinking in fast and slow pathways “ (Kahneman 2011). Zweiteres ist für das Erlernen der Kunstfer- 
tigkeit der Medizin unabdingbar. In diesen „slow pathways“ dominiert das Lust-/Unlustprinzip. Dieses „Denken“ ist ergänzend für die Verarbeitung von schwierigen Krankheitsprozessen unabdingbar, auch um eine Verarbeitungshilfe für den Patienten anbieten zu können ist ein Lernen und Denken in einem „slow pathway“ notwendig. Dies kann in der medizinischen Ausbildung in einer kontinuierlichen Lernerfahrung in einer Kleingruppe gemacht werden (vgl. Basiscurriculum Psychotherapie in der Psychiatriefacharztausbildung, Line-Elemente zu kommunikativen und professionellen Kompetenzen oder zum „scientific thinking“ im Medizincurriculum) oder mit professionellen Mentoren/Teaching-Professors, die für junge Menschen verantwortlich zeichnen. Diese müssten Identifikationsfiguren darstellen. Methodisch bietet sich eine leitfallorientierte Lehre an, ein fragengeleitetes Lernen entlang der Leitfälle, denn entlang von Prototypen lernt jeder Psychiater/jede Psychiaterin den klinischen Blick zu schärfen; mit den ersten prototypisch diagnostischen Erfahrungen, die der/die AusbildungskandidatIn im Leben gemacht hat, wird üblicherweise jeder weitere Fall abgeglichen.

So wie der Famulant in „Frost“ (Bernhard 1963) den Maler Strauch „offenbar im Zustand geistigen Verfalls“ beobachten soll, zuhören soll, den klinischen Blick schärfen soll, können wir junge Menschen an das Verstehen des „Außerfleischlichen“ heranführen, denn es geht ja darum, die künftige Generation $(Y, Z)$ in das Zuhören, in ein Verstehen Wollen, in das „Dahinrudern des Geistes hinzueinwerfen“, „einen Weg der Entdeckungen, einen solchen der nebeneinanderineinander-untereinander verlaufenden, miteinander korrespondierenden Anschauungsmöglichkeiten“ $\mathrm{zu}$ eröffnen, um den Blick für das Subjektive im Menschen zu schärfen.

\section{Konkrete Umsetzung}

Im Rahmen der interdisziplinären Diskussion wurde unter anderem dem Allgemeinmedizinischen Aspekt Raum gegeben, wobei hauptsächlich folgenden Fragen nachgegangen wurde: Welche Beobachtungen gibt es aus Sicht der Allgemeinmedizin und welche pragmatischen Umsetzungsmöglichkeiten ergeben sich für eine Kooperation der beiden Fachgruppen konkret. Dazu diskutierte Reinhold Glehr Präsident der Österreichischen Gesellschaft für Allgemein- und Familienmedizin:

Betont wurde hier die Beobachtung einer Zunahme der diagnostizierten psychischen Erkrankungen: zum einen beeinflusst eine veränderte Einstellung bzw. vermehrtes Wissen über psychische Erkrankungen der Menschen die zunehmende Diagnosehäufigkeit, jedoch ist auch eine absolute Zunahme wahrscheinlich. Als vermutliche Ursachen wurden folgende Beobachtungen diskutiert:

- Wandel in der Arbeitswelt und in anderen Lebenswelten insbesondere bedingt durch die neuen In- formations- und Kommunikationstechnologien in Beruf und Freizeit; wachsender Leistungsdruck Arbeitsverdichtung, schneller wechselnde Anforderungen, in Führungspositionen ständige Erreichbarkeit, Angst vor Arbeitslosigkeit.

- Destabilisierung sozialer Strukturen,

- größere Bereitschaft psychische Diagnosen zu akzeptieren,

- verbesserte hausärztliche diagnostische Kompetenz.

Weiters werden die Zunahme von Beschwerden mit psychischem Hintergrund bzw. eigentlichen psychischen Störungen (Weber et al. 2006; BARMER GEK 2015; DAK Forschung 2009), die Zunahme an Arbeitsunfähigkeitstagen aufgrund psychischer Diagnosen eine Verdopplung von 1976 bis 1991 in Deutschland, sowie ein nochmaliger Anstieg um 44\% nach 1991 (Dachverband Deutsche Betriebskrankenkassen 2008) und auch längere Ausfallzeiten (Falldauer mehr als 30 Tage) beobachtet.

Somit gewinnt die Allgemeinmedizin - als Einstiegsstelle und Begleiter eine spezifische Bedeutung, die zu einer Kooperation mit FachärztInnen für Psychiatrie und Psychotherapeutische Medizin auffordert: Beispielsweise zeigte eine Studie mit 1200 hausärztlichen Patienten (Glehr 2014) bei ca. $40 \%$ eine selbstvermutete psychische Krankheitskomponente, diejenigen PatientInnen mit psychischer Komponente waren kritischer und schwerer zur Entwicklung einer besseren Kompetenz für ihre Krankheitsbewältigung zu befähigen. Ältere, chronisch und multimorbid Kranke zeigten ein höheres Risiko für eine psychische Komponente. Des Weiteren sind 1/3 der im hausärztlichen Bereich vorgebrachten körperlichen Symptome mit psycho-sozialen Faktoren assoziiert (Dorner et al. 2010), auch wird die Prävalenz psychischer Störungen in der Allgemeinbevölkerung mit ca. 30 \% (Jacobi et al. 2004) angegeben.

In diesem Zusammenhang ist auch der Normbegriff der Krankheitsklassifikationen zugrunde liegt zu diskutieren, wie es auch in psychiatrisch-psychotherapeutischen Fachkreisen immer wieder geschieht. Seitens der Allgemeinmedizin wird ein stufenloser Übergang von gesund zu krank favorisiert: Konsultationen fordern „Diagnosen“ für den Nachweis der Leistungsberechtigung. In der Primärversorgung sind Symptome aber oft zu Beginn keiner abgrenzbaren Krankheit im Sinn einer Klassifizierung zuzuordnen. Dementsprechend ist die Grenze gesund-krank besonders bei psychischen Störungen fließend. Die in der Hausarztpraxishäufig vorliegende Frühsymptomatik mit allmählicher Näherung zur psychischen Komponente des Krankseins im hermeneutischen Sinn bringt in der Regel einen Zeitverzug bis zur definitiven Diagnose mit sich. Dies ist eine der möglichen Gründe für die schlechte Beurteilung allgemeinmedizinischer Kompetenz bei der Diagnostik psychischer Störungen in manchen Studien. 
Als weiterer wesentlicher Punkt wurde die Episodenhaftigkeit psychischer Störungen diskutiert: Die hausärztliche Beziehungs-Kontinuität ermöglicht ein Früherkennen von z. B. depressiven Episoden oder Exazerbationen von Psychosen. Eine Oligosymptomatik ist selten - nach simultanem Assessment somatischer und psychischer Probleme folgt häufig eine pragmatische Priorisierung/Fokussierung mit Gefahr der Ausblendung von Symptomen, die dann als neue Episode wiedererscheinen oder in anderen Bereichen des Gesundheitssystems sichtbar werden.

Aus all diesen Gründen ist die gestufte Versorgung - interdisziplinäre Versorgung gefordert. Die Notwendigkeit einer besseren, gegenseitig entlastenden $\mathrm{Zu}$ sammenarbeit zwischen ärztlichen Generalisten und Spezialisten, sowie nichtärztlichen Gesundheitsberufen unabdingbar und wird weltweit eingefordert! Eine gemeinsame Sprache, Therapieziele, abgestimmte Vorgehensweisen sind $\mathrm{zu}$ erarbeiten. Nationale Algorithmen zur gestuften, gemeinsamen Versorgung sind $\mathrm{zu}$ entwickeln (beispielsweise nach dem Vorbild des Mental Health Care Intervention Guide der WHO (World Health Organization 2011)). Eine verbesserte Ausbildung der Generalisten im psychischen Bereich ist notwendig, wie sie zum Teil in der ÄAO vorgesehen ist. Um die geplanten Kompetenzen und die dazu notwendigen Vermittlungsinstrumente bzw. -ressourcen zu operationalisieren, zu diskutieren und umzusetzen, wird eine gute Kooperation zwischen den Fächern Allgemeinmedizin und Psychiatrie und Psychotherapeutische Medizin erforderlich, zu welcher dieser Workshop einen Anstoß geben konnte.

Open access funding provided by Medical University of Vienna.

Interessenkonflikt H. Löffler-Stastka, B. Fink, K. MatuszakLuss, R. Glehr und A. Ruhs geben an, dass kein Interessenkonflikt besteht.

Open Access Dieser Artikel wird unter der Creative Commons Namensnennung 4.0 International Lizenz (http:// creativecommons.org/licenses/by/4.0/deed.de) veröffentlicht, welche die Nutzung, Vervielfältigung, Bearbeitung, Verbreitung und Wiedergabe in jeglichem Medium und Format erlaubt, sofern Sie den/die ursprünglichen Autor(en) und die Quelle ordnungsgemäß nennen, einen Link zur Creative
Commons Lizenz beifügen und angeben, ob Änderungen vorgenommen wurden.

\section{Literatur}

BARMER GEK (2015). Report Krankenhaus 2015. Schriftenreihe zur Gesundheitsanalyse, Bd.33, S.35-36).

Bernhard, T. (1963). Frost. Frankfurta. M.: Insel.

Bernhard, T. (1985). Die Kälte. Eine Isolation (2. Aufl.). München:Deutscher Taschenbuch Verlag. Erstausgabe 1981

Bernhard, T. (2010). Ein Kind (Autobiographische Schriften. Bd.5.Salzburg: Residenz-Verlag. Erstausgabe 1982

Bernhard, T. (2014). Der Atem. Eine Entscheidung (2. Aufl.). München: Deutscher Taschenbuch Verlag. Erstausgabe 1978

Bion, W. R. (1967). Second thoughts: selected papers on psychoanalysis. London: Karnac.

Dachverband Deutsche Betriebskrankenkassen (2008). BKK Gesundheitsreport 2008(S. 12-17).

DAK Forschung (2009). DAK Gesundheitsreport 2009 (S. 7). Berlin: IGESInstitut.

Dorner, T.E., Stronegger, W.J., Rebhandl, E., Rieder, A., \& Freidl, W. (2010). The relationship between various psychosocial factors and physical symptoms reported during primary-care health examinations. Wien Klin Wochenschr, 122(3-4), 103-109.

Fonagy, P., Gergely, G., Jurist, E., \& Target, M. (2002). Affect Regulation, Mentalization and the Development of the Self. New York: Other Press.

Freud, S. (1907). Der Wahn und die Träume. In W. Jensens (Hrsg.), Gradiva (S.9-85). Stud.X.

Glehr, R. (2014). The influence of a psychosocial training on the quality of consultations in primary care in Austria (S. 37). Wien: Institut für Allgemein- und Familienmedizin, Medizinische UniversitätWien.

Jacobi, F., Klose, K., \& Wittchen, H.-U. (2004). Psychische Störungen in der deutschen Allgemeinbevölkerung: Inanspruchnahme von Gesundheitsleistungen und Ausfalltage. Bundesgesundheitsblatt, 47, 736-744. doi:10. 1007/s00103-004-0885-5

Kahneman, D. (2011). Thinking, fast and slow. London: Penguin.

Watkins, J. G., \& Watkins, H.H. (2008). Ego State-Theorie und Therapie. Ein Handbuch (2. Aufl.). Heidelberg: Carl Auer.

Weber, A., Hörmann, G., \& Köllner, V. (2006). Psychische und Verhaltensstörungen:DieEpidemie des21.Jahrhunderts? Dtsch Arztebl, 103(13), A-834/B-712-C-688.

World Health Organization (2011). Intervention Guide for mental, neurological and substance use disorders in nonspecialized health settings. ISBN 9789241548069. 